\title{
Factores cognitivo-motivacionales relacionados con el ajuste a la vida universitaria de estudiantes chilenos
}

\section{Cognitive-motivational factors related to adjustment to university life of Chilean students}

\author{
Maite Zuñiga-Vilches ${ }^{1}$, Jorge Vergara-Morales ${ }^{2}$, \\ María-Victoria Pérez-Villalobos ${ }^{1}$, Alejandro Díaz-Mujica ${ }^{1}$ \\ 'Universidad de Concepción, Chile \\ 2Universidad de Las Américas, Chile
}

\section{Resumen}

Los desafíos que implica la adaptación a la vida universitaria pueden constituir una sobrecarga de recursos personales relacionados con aspectos académicos y cognitivo-motivacionales, lo que puede afectar la decisión de continuar los estudios universitarios. Por este motivo, el objetivo del estudio fue evaluar la relación del aprendizaje autorregulado y la autoeficacia académica con el ajuste a la vida universitaria, considerando el rol mediador de la autorregulación del aprendizaje de estudiantes universitarios chilenos. Se utilizó un diseño cuantitativo, no experimental y transversal, considerando una estrategia asociativa de carácter explicativa. Los participantes fueron un total de 470 estudiantes de universitarios. Los datos se analizaron mediante estadística descriptiva, análisis de correlación y análisis de mediación SEM. Los resultados de correlación mostraron que las relaciones variaron en su magnitud de acuerdo con las dimensiones del ajuste a la vida universitaria. Además, se identificó que la autorregulación del aprendizaje tiene un rol mediador en el efecto de la autoeficacia académica sobre el ajuste a la vida universitaria. Estos hallazgos permiten afirmar la importancia que tienen las variables socio-cognitivas en el proceso de adaptación de estudiantes universitarios.

Palabras clave: Autorregulación del aprendizaje, autoeficacia académica, ajuste a la vida universitaria, análisis de mediación

\begin{abstract}
The challenges for adapting to university life may constitute an overload of personal resources related to academic and cognitive-motivational aspects, which may affect the decision to continue university studies. For this reason, the aim of the research was to assess the relationship of the self-regulated learning and academic self-efficacy with the adjustment to university life, considering the mediating role of the self-regulated learning of Chilean university students. A quantitative, non-experimental, and cross-sectional design was used, considering an explanatory associative strategy. Data were analyzed using descriptive statistics, correlation analysis, and SEM mediation analysis. The participants were a total of 470 university students. The correlation results showed that the relationships varied in magnitude according to the dimensions of adjustment to university life. Moreover, it was identified that the self-regulated learning has a mediating role in the effect of academic self-efficacy on the adjustment to university life. These findings allow us to affirm the importance of socio-cognitive variables in the adaptation process of university students.
\end{abstract}

Keywords: Self-regulated learning, academic self-efficacy, adjustment to university life, mediation analysis

Cómo citar este artículo: Zuñiga-Vilches, M., Vergara-Morales, J., Pérez-Villalobos, M-V., \& Díaz-Mujica, A. (2020). Factores cognitivo-motivacionales relacionados con el ajuste a la vida universitaria de estudiantes chilenos. Escritos de Psicología - Psychological Writings, 13(2), 71-79. https://doi.org/1024310/espsiescpsi.v13i2.12411

Correspondencia: Jorge Vergara-Morales. Facultad de Salud y Ciencias Sociales, Escuela de Psicología. Chacabuco 539. CONCEPCIÓN, CHILE. E-mail: jvergaram@udla.cl. Coautores/as: Maite Zuñiga-Vilches: mzunigav@udd.cl, María-Victoria Pérez: marperez@udec.cl, Alejandro Díaz: adiazm@udec.cl 


\section{Introducción}

Durante el desarrollo de la vida universitaria, los estudiantes están expuestos a numerosos procesos de adaptación y desafíos que pueden constituirse como situaciones estresantes (Fernández, Araújo, Vacas, Almeida, \& González, 2017; Riquelme et al., 2012). En comparación con las actividades de la educación secundaria, en la universidad son menos continuadas, los horarios son más flexibles, existe menos control, la relaciones entre profesores y alumnos tienden a ser más distantes, las exigencias académicas son más complejas y existe una mayor heterogeneidad entre los compañeros de clase. Esto implica dificultades diversas para el establecimiento de relaciones de amistad o apoyo social en el nuevo ambiente académico (Soares et al., 2014).

La transición a la universidad exige un nivel adecuado de competencias personales, tales como la madurez, la autonomía y la capacidad de adaptación para hacer frente a esta nueva etapa en la vida académica de los estudiantes (Fernández et al., 2017; Soares et al., 2014). El periodo de transición a la universidad y los desafíos que conlleva, suponen para muchos estudiantes una sobrecarga a sus recursos personales, lo que puede producir dificultades de ajuste a la vida universitaria (Fernández et al., 2017), generando consecuencias negativas relacionadas con un bajo desempeño académico y el abandono de los estudios superiores (Fernández et al., 2017; van Rooij, Jansen, \& van de Grift, 2017).

\section{Ajuste a la vida universitaria}

El ajuste a la vida universitaria, es entendido como la capacidad que tiene un estudiante para adaptarse a las demandas del contexto universitario (Baker \& Siryk, 1984, 1986)yielding indices of 4 aspects of adjustment as well as a full-scale score, was developed and administered once each semester in each of 3 successive academic years to samples of freshman students $(N=734$. Se trata de un proceso de múltiples facetas que exige la adaptación a la cultura, normas y valores de la institución académica escogida por el estudiante (Ambiel, Santos \& Dalbosco, 2016). Exige que el estudiante asuma una actitud responsable y proactiva para resolver las diversas situaciones que son propias del periodo de transición y necesarias para aprobar los cursos exitosamente. Además, se requiere que el estudiante utilice recursos personales, interpersonales y familiares para alcanzar un ajuste sano al contexto universitario (Ambiel, Santos \& Dalbosco, 2016). Supone la consolidación de la propia identidad y una adecuada adaptación a las situaciones académicas que incluyen los ámbitos: personal, interpersonal, de la carrera y asociada a los estudios (Ambiel et al., 2016; Soares et al., 2014).

Una medida de ajuste global considera los siguientes aspectos: (1) ajuste personal - emocional, vinculado al estado psicológico del estudiante y los niveles de angustia experimentados; (2) ajuste académico, referido a la motivación por cumplir los requisitos académicos, el esfuerzo y la adaptación al ambiente universitario; (3) ajuste social, entendido como el nivel de éxito que alcanza un estudiante para relacionarse en contextos interpersonales inherentes al contexto universitario; y (4) ajuste institucional, referido a la satisfacción del estudiante con su centro educativo y la calidad de la relación entre la universidad y el estudiante (Baker \& Siryk, 1986)for comparison through interview, students occupying extremes of score distributions on measures of effectiveness of adjustment to college, (b.

\section{Autorregulación del aprendizaje}

En el análisis de los factores que determinan el éxito en la educación superior, se ha encontrado que los factores personales son determinantes para obtener un buen desempeño académico, destacándose el uso de estrategias de aprendizaje autorregulado (Fernández et al., 2013). Desde la perspectiva socio-cognitiva, la autorregulación del aprendizaje se considera un proceso de origen social, mediante el cual los estudiantes establecen sus metas de aprendizaje, monitorean, regulan y controlan su cognición, emociones, motivaciones y conducta, en función de metas y características contextuales (Fernández et al., 2013; Lamas Rojas, 2008; Panadero \& Alonso-Tapia, 2014a, 2014b; Pérez \& Gutierrez-Braojos, 2012; Rosário et al., 2014; Zimmerman, 1989; Zulma Lanz, 2006). Esto supone que el desarrollo de la competencia autorreguladora no es resultado exclusivo de la maduración de los individuos, ni tampoco un proceso que emerja a partir del ambiente (Pérez \& Gutierrez-Braojos, 2012; Zimmerman, 1989). Se trata del resultado de un proceso que integra factores comportamentales, ambientales y personales (cognición y emoción) recíprocamente determinados (Pérez \& Gutierrez-Braojos, 2012). En esta línea, se ha propuesto un modelo explicativo conformado por tres fases cíclicas, íntimamente relacionadas entre sí, que están en constante interacción: (a) la planificación de la tarea, (b) la ejecución, y (c) evaluación de la misma (Panadero \& Alonso-Tapia, 2014b; Pérez \& Gutierrez-Braojos, 2012; Zimmerman, 2008). 


\section{Autoeficacia académica}

La autoeficacia académica se ha constituido como uno de los constructos más reconocidos para predecir el desempeño y el éxito académico (Salanova, Martínez, \& Llorens, 2012; van Rooij et al., 2017), dado que determina el tiempo y el esfuerzo que el estudiante dedica a una determinada tarea, contribuyendo significativamente a la motivación, bienestar y desempeño académico (Salanova et al., 2012). La autoeficacia es definida como los juicios sobre la propia capacidad para alcanzar determinados niveles de rendimiento (Bandura, 1977). Se entiende como el sistema de creencias respecto de la propia capacidad para desempeñarse en el ámbito académico. Se trata de juicios que el estudiante realiza acerca de su propia capacidad y los recursos con los que cuenta para conseguir exitosamente sus objetivos académicos, condicionando el inicio y la persistencia de una determinada actividad académica (Ornelas, Blanco, Gastélum, \& Chávez, 2012).

Los juicios que los estudiantes poseen acerca de la propia capacidad, se constituyen a partir de cuatro fuentes de información: experiencias personales directas, experiencias vicarias, procesos sociales y procesos de inferencia, siendo la primera de ellas la que mejor predice la autoeficacia (Bartimote-Aufflick et al., 2016).

\section{Relación entre variables}

Estudios previos han evidenciado una relación entre el ajuste a la vida universitaria, la autoeficacia académica (Norwalk, Norvilitis, \& MacLean, 2009) y el aprendizaje autorregulado (Cazan, 2012). En la relación con esta última variable, se ha destacado especialmente con la dimensión de ajuste académico, evidenciándose incluso una capacidad predictiva del aprendizaje autorregulado sobre el ajuste académico de los estudiantes (Cazan, 2012; Cazan \& Anitei, 2010). Es decir, se ha observado que estudiantes que dedican tiempo a planificar sus actividades académicas, son conscientes de sus métodos de estudio, evalúan sus resultados académicos y analizan sus acciones para mejorar, tiene una mejor adaptación a las demandas académicas de la universidad, aún en situaciones estresantes, ya que las estrategias de autorregulación favorecen la asignación de recursos de manera eficiente y la búsqueda oportuna de ayuda cuándo es necesario (Cazan,2012; Cazan y Anitei, 2010; van Rooij et al., 2017).

La autoeficacia académica también se ha asociado de forma importante al ajuste a la vida universitaria (Chen, Solberg, \& Ye, 2018), especialmente en estudiantes de primer año de universidad, en dónde la autoeficacia se comporta como predictor de ajuste académico (Cazan, 2012). Así, los estudiantes que se perciben con altas capacidades académicas, que tienen un alto grado de confianza en su capacidad para aprender y obtener éxito académico tienen más probabilidades de hacer frente a las demandas académicas de la universidad y lograr cumplir los requisitos para aprobar sus asignaturas (Chen et al., 2018; Cazan, 2012).

El ajuste a la vida universitaria se constituye como un importante predictor de éxito académico y retención en educación superior (Fernández et al., 2017). Tal cómo se ha mencionado en los párrafos anteriores, las investigaciones previas sugieren una relación positiva e incluso de carácter predictivo de las variables cognitivo-motivacionales estudiadas sobre el ajuste a la vida universitaria.

En relación con el rol mediador de la autorregulación del aprendizaje, los estudios han mostrado la capacidad de intervenir el efecto de factores cognitivos y motivacionales en el proceso de aprendizaje (Ariani, 2016; Jansen, van Leeuwen, Janssen, Jak \& Kester, 2019; Lee, Moon \& Cho, 2015). Debido al impacto positivo que tiene el ajuste a la vida universitaria en el éxito académico y la persistencia en los estudios universitarios, es que se considera relevante estudiar las dimensiones de la variable ajuste a la vida universitaria y su relación con el aprendizaje autorregulado y la autoeficacia académica, dado que las variables cognitivas y motivacionales son susceptibles de ser modificadas e influenciadas en la interacciones propias del contexto académico, especialmente en la relación profesor-estudiante (Díaz, 2008). Con este estudio se busca contribuir con información acerca de estas variables que afectan el ajuste de estudiantes universitarios, lo cual puede influir en la decisión de abandonar los estudios universitarios, problema que se ha consolidado como prioritario en los últimos treinta años para el ámbito educativo a nivel nacional e internacional (Rivera, Martínez, González \& Salazar, 2016).

Por lo tanto, el objetivo del estudio es evaluar la relación del aprendizaje autorregulado y la autoeficacia académica con el ajuste a la vida universitaria, considerando el rol mediador de la autorregulación del aprendizaje de estudiantes universitarios chilenos. Como hipótesis de investigación, se plantea que la autoeficacia académica tiene un efecto indirecto sobre el ajuste a la vida universitaria a través de la autorregulación del aprendizaje. Más específicamente, se plantea que la autorregulación del aprendizaje tiene un efecto mediador parcial en la relación entre la autoeficacia académica y el ajuste a la vida universitaria. 


\section{Diseño}

\section{Método}

El estudio se orientó desde un diseño cuantitativo, no experimental y transversal. Además, se utilizó una estrategia asociativa de tipo explicativa, ya que se evaluaron modelos de relaciones basados en perspectivas teóricas subyacentes (Ato, López \& Benavente, 2013).

\section{Participantes}

Los participantes fueron un total de 470 estudiantes de primer año de una universidad chilena, pertenecientes a carreras del área de la Ingeniería. El 68,9\% fueron participantes del género masculino (n $=324$ ) y el $31,1 \%$ fueron participantes del género femenino $(n=146)$. La edad varió de 17 a 33 años, con una edad media de 18.81 años $(D E=1.42)$. La selección de los participantes se realizó mediante un muestreo accidental, teniendo como criterios de inclusión el cursar por primera vez, el primer año académico de una carrera de pregrado.

\section{Instrumentos}

Cuestionario de Adaptación a la Vida Universitaria (Baker \& Siryk, 1989). Este cuestionario evalúa cómo los estudiantes se ajustan a la universidad (Rodríguez, Tinajero, Guisande, \& Páramo, 2012). Se realizó una adaptación para estudiantes universitarios chilenos que contiene 43 reactivos, con una escala de respuesta tipo Likert de 7 puntos ( 1 = totalmente en desacuerdo; $7=$ totalmente de acuerdo). Los ítems se distribuyen en cuatro factores: (a) ajuste académico, (16 ítems, ej. "Últimamente no he sido eficaz con el uso de mi tiempo de estudio"); (b) ajuste social (12 ítems, ej. "Creo que encajo bien en la Universidad"); (c) ajuste personal-emocional (12 ítems, ej. "Últimamente he experimentado tristeza y bastantes cambios de humor"); y (d) apego institucional (9 ítems, ej. "Estoy satisfecho(a) de estar en esta Facultad"). En el presente estudio los factores presentaron adecuados niveles de consistencia interna ( $\alpha=0.79$ para ajuste académico, $\alpha=0.84$ para ajuste social, $\alpha=0.86$ para ajuste emocional $y$ $\alpha=0.82$ para ajuste institucional).

Inventario de Procesos de Autorregulación del Aprendizaje (IPAA) (Bruna, Pérez, Bustos, \& Núñez, 2017). Este instrumento evalúa las estrategias de autorregulación del aprendizaje utilizadas por los estudiantes universitarios. Se compone de 12 ítems organizados en 3 dimensiones representativas de las fases del proceso de autorregulación del aprendizaje propuestas por Zimmerman (2002): (a) planificación (5 ítems, ejemplo: "hago un plan antes de comenzar a hacer un trabajo escrito, pienso lo que voy a hacer y lo que necesito para conseguirlo"; $\alpha=0.68$ ); (b) ejecución (3 ítems, ejemplo: "cuando estudio, trato de comprender la materia, tomar apuntes, hacer resúmenes, resolver ejercicios o hacerme preguntas sobre los contenidos", $\alpha=0.66$ ) y (c) evaluación ( 4 ítems, ejemplo: "guardo y analizo las correcciones de los trabajos escritos o pruebas parciales, para saber dónde me equivoqué y saber qué tengo que cambiar para mejorar", $\alpha=0.59)$. La puntuación global de la escala presentó un nivel adecuado de consistencia interna $(\alpha=0.81)$. Los ítems se respondieron en una escala de respuesta tipo Likert de 7 puntos ( 1 = nunca; 7 = siempre). En esta investigación, la puntuación global de la escala presentó un adecuado nivel de fiabilidad $(\alpha=.87)$.

Escala de Autoeficacia Percibida Específica de Situaciones Académicas (García-Fernández et al., 2010). Se compone de 10 ítems que miden las expectativas de autoeficacia en situaciones específicas del contexto educativo universitario (p.ej.: "Tengo la convicción de que puedo obtener excelentes notas en las pruebas"). En este estudio, los ítems fueron evaluados en una escala de respuesta tipo Likert de 7 puntos ( 1 = totalmente en desacuerdo; 7 = totalmente de acuerdo). La suma total de las puntuaciones indicó el grado de autoeficacia académica percibida por los estudiantes. En estudiantes chilenos, las puntuaciones de la escala han presentado un nivel adecuado de consistencia interna $(\alpha=.88)$, manteniendo la estructura unifactorial propuesta en la versión original (Palenzuela, 1983). En esta investigación, las puntuaciones de la escala presentaron un alto nivel de fiabilidad $(\alpha=.92)$.

\section{Procedimiento}

Se estableció contacto con los/as directores/as de cada una de las carreras participantes, explicando los objetivos, contexto, alcances y limitaciones del estudio, declarando el resguardo de la confidencialidad de los participantes. Además, se realizó la coordinación del proceso de aplicación de los instrumentos, los que fueron aplicados en la sala de clases, considerando un tiempo aplicación aproximado de 20 minutos. Los participantes fueron informados del carácter voluntario y confidencial de su participación, teniendo la libertad de abandonar el estudio en cualquier momento sin que esto tuviese consecuencias negativas para ellos. Esta información se le dio a conocer de manera verbal y escrita mediante consentimiento informado. 
Análisis de los datos

En primer lugar, se realizó el análisis descriptivo calculando la media, la desviación estándar y los valores de asimetría y curtosis, considerando un punto de corte de \pm 3 para indicar valores aceptables (Ryu, 2011). Además, se utilizó el coeficiente de correlación de Pearson para evaluar el grado de asociación entre las variables de estudio. Posteriormente, el análisis de mediación se realizó mediante modelación de ecuaciones estructurales, utilizando el método de estimación de máxima verosimilitud (ML). La bondad de ajuste del modelo de medida se evaluó considerando los siguientes índices y criterios: chi-cuadrado $\left(X^{2}\right)$ y chi-cuadrado normalizado $\left(X^{2} / d f\right)=$ valores menores a 2 (Ullman, 2001) y menores a 5 (West et al., 2012) se consideran aceptables; Índice de Tucker Lewis (TLI); Índice de ajuste comparativo $(\mathrm{CFI})=$ valores $\geq 0.90$ evidencian un ajuste aceptable y valores $\geq 0.95$ indican un buen ajuste; Error cuadrático medio de aproximación (RMSEA) y raíz cuadrática media estandarizada residual $(S R M R)=$ los valores $\leq 0.50$ indican un buen ajuste y los valores $\leq 0.80$ indican un ajuste aceptable (Schweizer, 2010). Finalmente, se utilizó el procedimiento bootstrapping con 5000 iteraciones para evaluar los efectos directos e indirectos, los que se consideraron estadísticamente significativos cuando las estimaciones del intervalo de confianza del 95\% no contenían el valor cero. Los análisis se realizaron utilizando los programas SPSS versión 21 (IBM Corporation, 2012) y Mplus versión 8 (Muthén y Muthén, 2012).

\section{Análisis descriptivo}

\section{Resultados}

En la tabla 1, se observa que las puntuaciones medias evidenciaron niveles positivos de ajuste a la vida universitaria, autorregulación del aprendizaje y autoeficacia académica. Al respecto, se observa que el ajuste institucional $(M=6.25)$ y el ajuste social $(M=5.36)$ presentaron las puntuaciones medias más altas. Por otro lado, el ajuste académico $(M=4.92)$ y el ajuste emocional $(M=4.10)$ presentaron las puntuaciones medias más bajas. Además, se identifica que los datos presentaron niveles adecuados de dispersión, ya que las puntuaciones se situaron cercanas a 1.00. Finalmente, las medidas de asimetría y curtosis evidencian una tendencia hacia la distribución normal univariada, ya que la mayoría de las puntuaciones se situaron en el límite \pm 3.00 .

Tabla 1

\begin{tabular}{lcccc} 
Estadísticos descriptivos & \multicolumn{3}{l}{} \\
\hline Variables & $\boldsymbol{M}$ & $\boldsymbol{D E}$ & Asimetría & Curtosis \\
\hline Ajuste institucional & 6.25 & 0.80 & -1.61 & 3.08 \\
Ajuste académico & 4.92 & 0.81 & -0.03 & -0.54 \\
Ajuste social & 5.36 & 0.96 & -0.60 & -0.28 \\
Ajuste emocional & 4.10 & 1.20 & -0.08 & -0.70 \\
Autorregulación del aprendizaje & 5.06 & 1.00 & -0.53 & 0.20 \\
Autoeficacia académica & 5.07 & 1.00 & -0.61 & 0.26 \\
\hline
\end{tabular}

Análisis de correlación

En la tabla 2, se muestra que las variables de estudio se asociaron de manera positiva y significativa. Respecto de la autorregulación del aprendizaje, se observa que las asociaciones más fuertes se presentaron con el ajuste académico $(r=.50, p<.01)$ y el ajuste institucional $(r=.32, p<.01)$. Por otro lado, se identifica que la autoeficacia académica presentó las asociaciones más fuertes con el ajuste académico $(r=.54, p<.01)$ y el ajuste emocional $(r=.42, p<.01)$.

Tabla 2

Correlaciones entre variables

\begin{tabular}{lcc}
\hline Ajuste a la vida universitaria & Autorregulación del aprendizaje & Autoeficacia académica \\
\hline Ajuste institucional & $.32^{* *}$ & $.31^{* *}$ \\
Ajuste académico & $.50^{* *}$ & $.54^{* *}$ \\
Ajuste social & $.29^{* *}$ & $.38^{* \star}$ \\
Ajuste emocional & $.25^{* *}$ & $.40^{* *}$ \\
\hline Nota ${ }^{* *} p<0.01$ & &
\end{tabular}

\section{Análisis de mediación}

El modelo propuesto incluyó tres factores latentes y 26 indicadores (ver Figura 1). Al respecto, se consideró un factor que mide el ajuste a la vida universitaria mediante cuatro indicadores, otro factor que mide la autorregulación del aprendizaje con doce indicadores, y un factor que mide la autoeficacia académica mediante diez indicadores. Los resultados mostraron que el modelo de mediación simple 
cumple con los criterios recomendados y evidencia un ajuste aceptable a los datos observados, explicando el $33 \%$ de la varianza de la autorregulación del aprendizaje y el $49 \%$ de la varianza del ajuste a la vida universitaria: $\mathrm{X}^{2}=860.74 ; \mathrm{X}^{2} / \mathrm{gl}=2.92 ; \mathrm{TLI}=0.90 ; \mathrm{CFI}=0.91 ; \mathrm{RMSEA}=0.06, \mathrm{SRMR}=0.06$.

A partir del ajuste aceptable del modelo de mediación testeado, se calcularon los efectos directos e indirectos. En primer lugar, la autoeficacia académica se relacionó positiva y significativamente con la autorregulación del aprendizaje [a $=0.59, \mathrm{SE}=0.06, p<0.01]$. En segundo lugar, la autorregulación del aprendizaje se relacionó positiva y significativamente con el ajuste a la vida universitaria, después de controlar por la autoeficacia académica $[b=0.23, S E=0.05, p<0.01]$. En tercer lugar, la autoeficacia académica se relacionó de forma significativa e indirecta con el ajuste a la vida universitaria a través de la autorregulación del aprendizaje $[\mathrm{ab}=0.13, \mathrm{SE}=0.03,95 \% \mathrm{Cl}(0.09 \div 0.19), \mathrm{p}<0.01]$. Debido a que el efecto directo de la autoeficacia académica continúa siendo significativo [c' $=0.34, \mathrm{SE}=0.04, \mathrm{p}$ $<0.01$ ], se puede concluir que la autorregulación del aprendizaje media parcialmente la relación entre la autoeficacia académica y el ajuste a la vida universitaria.

Figura 1

Modelo de mediación simple

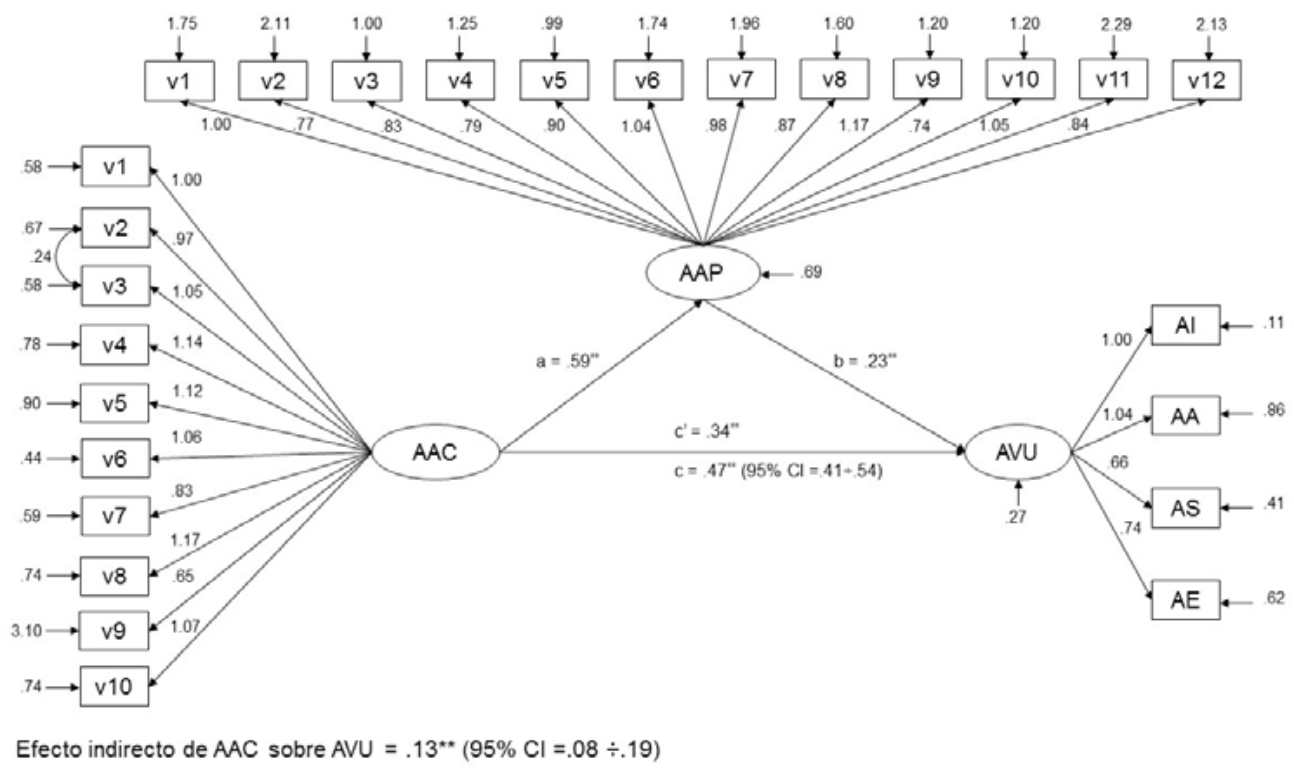

$A A C=$ Autoeficacia académica; $A A P=$ Autorregulación del aprendizaje; $A V U$ = Ajuste a la vida universitaria; $A I=$ Ajuste institucional; $A A=$ Ajuste académico; $A S=$ Ajuste social; $A E$ = Ajuste emocional; ${ }^{* *} p<.01$; coeficientes no estandarizados

\section{Discusión y conclusiones}

El presente estudio tuvo como objetivo evaluar la relación de la autorregulación del aprendizaje y la autoeficacia académica con el ajuste a la vida universitaria, considerando el rol mediador de la autorregulación del aprendizaje de estudiantes universitarios chilenos. Los resultados muestran que existe una relación positiva y significativa entre las diferentes dimensiones del ajuste a la vida universitaria con las variables sometidas a estudio, tal como ha sido indicado en los hallazgos de Caballero, Abello, \& Palacio (2007) y van Rooij et al. (2017). Estos resultados permiten afirmar la relevancia que tienen las variables de estudio en la configuración del ajuste a la vida universitaria. Al respecto, mientras se observan altas capacidades para establecer metas y regular el proceso de aprendizaje, se presentan principalmente altas capacidades de ajuste académico en los estudiantes. Por otro lado, mientras se observa una percepción favorable sobre la capacidad para alcanzar las metas, se identifican principalmente altas capacidades de ajuste académico y emocional. De esta manera, se concluye que los grados de asociación de la autorregulación del aprendizaje y la autoeficacia académica con el ajuste a la vida universitaria varían en su magnitud de acuerdo a las dimensiones específicas del constructo. Esto permite delimitar la forma en que cada una de las variables puede contribuir al proceso de adaptación al contexto de educación superior.

Además, se identificó que la autoeficacia académica tiene un efecto indirecto sobre el ajuste a la vida universitaria a través de la autorregulación del aprendizaje percibida por los estudiantes. Más específicamente, se observó que la autorregulación del aprendizaje tuvo un efecto mediador parcial 
sobre la relación entre la autoeficacia académica y el ajuste a la vida universitaria, lo que confirma la hipótesis de investigación propuesta. Estos resultados coinciden con los hallazgos del trabajo realizado por Lee et al. (2015), lo que pone de manifiesto el importante rol que tiene el aprendizaje autorregulado de los estudiantes en el proceso de adaptación a las demandas del contexto universitario, ya que no sólo influye positivamente en el ajuste a la vida universitaria, sino que también contribuye a explicar la relación entre la autoeficacia académica y el proceso de adaptación de los estudiantes al contexto universitario, debido a que el desarrollo de la competencia autorreguladora en el proceso de aprendizaje promueve la influencia que los juicios sobre la propia capacidad para el logro de los aprendizajes ejercen en el ajuste de los estudiantes a la vida universitaria.

Debido a que el ajuste a la vida universitaria constituye un factor clave para que el alumnado continúe sus estudios y se logre la meta de graduación, es necesario que las instituciones universitarias desarrollen planes de intervención específicos que promuevan el desarrollo y fortalecimiento de la autorregulación del aprendizaje, ya que resulta fundamental para el ajuste a la vida universitaria de los estudiantes. En este sentido, resulta fundamental la generación de estrategias que faciliten la incorporación de la autorregulación del aprendizaje en el ámbito de los diseños curriculares y las actividades pedagógicas que realizan los docentes universitarios.

Dentro de las limitaciones del estudio, es importante mencionar que los participantes fueron estudiantes universitarios de primer año. Por lo tanto, es importante que futuras investigaciones consideren una muestra más heterogénea que permita ampliar la validez de los hallazgos. Por otro lado, el estudio se realizó desde un diseño transversal, lo que dificulta la interpretación de la inferencia causal de los resultados. Por lo tanto, es importante que los estudios posteriores consideren diseños longitudinales de investigación.

\section{Notas de autor.}

Agradecimientos al Proyecto ANID-COVID1012

\section{Referencias}

1. Ambiel, R. A. M., Santos, A. A. A. d., \& Dalbosco, S. N. P. (2016). Motivos para evasão, vivências acadêmicas e adaptabilidade de carreira em universitários. Psico-USF, Bragança Paulista, 47(4), 288-297. https://doi.org/10.15448/1980-8623.2016.4.23872

2. Ariani, D. W. (2016). Why do I study? The mediating effect of motivation and self-regulation on student performance. Business, Management and Education, 14, 153-178. https://doi.org/10.3846/ bme.2016.329

3. Ato, Manuel, López, Juan J., \& Benavente, Ana (2013). Un sistema de clasificación de los diseños de investigación en psicología. Anales de Psicología, 29,1038-1059. https://doi.org/10.6018/ analesps.29.3.178511

4. Bartimote-Aufflick, K., Bridgeman, A., Walker, R., Sharma, M., \& Smith, L. (2016). The study, evaluation, and improvement of university student self-efficacy. Studies in Higher Education, 41, 1918-1942. https://doi.org/10.1080/03075079.2014.999319

5. Baker, R., \& Siryk, B. (1984). Measuring Adjustment to College. Journal of Counseling Psychology, 31, 179-189. https://doi.org/10.1037/0022-0167.31.2.179

6. Baker, R., \& Siryk, B. (1986). Exploratory intervention with a scale measuring adjustment to college. Journal of Counseling Psychology, 33, 31-38. https://doi.org/10.1037//0022-0167.33.1.31

7. Bandura, A. (1977). Self-efficacy: Toward a unifying theory of behavioral change. Psychological Review, 84, 191-215. https://doi.org/10.1037/0033-295x.84.2.191

8. Bruna, D., Pérez, M. V., Bustos, C., \& Núñez, J. C. (2017). Propiedades Psicométricas del Inventario de Procesos de Autorregulación del Aprendizaje en Estudiantes Universitarios Chilenos. Revista Iberoamericana de Diagnóstico y Evaluación Psicológica, 44, 77-91. https://doi. org/10.21865/RIDEP44.2.07

9. Caballero, C. C. D., Abello, R. L. L., \& Palacio, S. Y. J. (2007). Relationship between burnout, academic berformance, and satisfaction concerning study, in college students. Avances en Psicologia Latinoamericana, 25, 98-111.

10. Cazan, A. M. (2012). Self-regulated learning strategies - Predictors of academic adjustment. Procedia - Social and Behavioral Sciences, 33, 104-108. https://doi.org/10.1016/j.sbspro.2012.01.092

11. Cazan, A. M., \& Anitei, M. (2010). Motivation, learning strategies and academic adjustment. Romanian Journal of Experimental Applied Psychology, 1, 61-69. 
12. Chen, Z., Solberg, S., \& Ye, A. (2018). Chinese Youth Career Adaptability: Contextual Influences and Pathways to Positive Youth Development. Youth \& Society, 52, 1-26. https://doi. org/10.1177/0044118x18784058

13. Díaz, C. (2008). Modelo conceptual para la deserción estudiantil universitaria chilena. Estudios pedagógicos (Valdivia), 34(2), 65-86. https://doi.org/10.4067/S0718-07052008000200004

14. Fernández, E., Bernardo, A., Suárez, N., Cerezo, R., Núñez, J. C., \& Rosario, P. (2013). Predicción del uso de estrategias de autorregulación en educación superior. Anales de Psicología, 29, 865-875. https://doi.org/10.6018/analesps.29.3.139341

15. Fernández, M. F. P., Araújo, A. M., Vacas, C. T., Almeida, L. S., \& González, M. S. R. (2017). Predictors of students' adjustment during transition to university in Spain. Psicothema, 29, 67-72. https://doi.org/10.7334/psicothema2016.40

16. García-Fernández, J. M., Inglés, C. J., Torregrosa, M. S., Ruiz-Esteban, C., Díaz-Herrero, A., PérezFernández, E., \& Martínez-Monteagudo, M. C. (2010). Propiedades psicométricas de la Escala de Autoeficacia Percibida Específica de Situaciones académicas en una muestra de estudiantes españoles de Educación Secundaria Obligatoria. European Journal of Educational Psychology, 3, 61-74. Recuperado de http://www.redalyc.org/articulo.oa?id=129313736005

17. IBM Corporation (2012). IBM SPSS Statistics for Windows, Version 21.0. Armonk, NY: IBM Corp.

18. Jansen, R. S., van Leeuwen, A., Janssen, J., Jak, S., \& Kester, L. (2019). Self-regulated learning partially mediates the effect of self-regulated learning interventions on achievement in higher education: A meta-analysis. Educational Research Review, 28, 100292. https://doi.org/10.1016/j. edurev.2019.100292

19. Lamas Rojas, H. (2008). Aprendizaje autorregulado, motivación y rendimiento académico. Liberabit, 14, 15-20.

20. Lee, J., Moon, J., \& Cho, B. (2015). The Mediating Role of Self-Regulation Between Digital Literacy and Learning Outcomes in the Digital Textbook for Middle School English. Educational Technology International, 16, 58-83. Recuperado de http://kset.or.kr/eti_ojs/index.php/instruction/ article/viewFile/34/pdf 9

21. Muthen, L. K., \& Muthen, B. (2012). 1998-2012. Mplus User's Guide (7th ed.). Los Angeles, CA: Muthen \& Muthen.

22. Norwalk, K., Norvilitis, J. M., \& MacLean, M. G. (2009). ADHD symptomatology and its relationship to factors associated with college adjustment. Journal of Attention Disorders, 13, 251-258. https://doi.org/10.1177/1087054708320441

23. Ornelas, M., Blanco, H., Gastélum, G., \& Chávez, A. (2012). Perceived self-efficacy in the academic behavior of female university students. Formación Universitaria, 5, 17-26. https://doi. org/10.4067/S0718-50062012000200003

24. Palenzuela, D. (1983). Construcción y validación de una escala de autoeficacia percibida específica de situaciones académicas. Análisis y Modificación de Conducta, 9, 185-219.

25. Panadero, E., \& Alonso-Tapia, J. (2014a). Teorías de autorregulación educativa: una comparación y reflexión teórica. Psicología Educativa, 20(1), 11-22. https://doi.org/10.1016/j.pse.2014.05.002

26. Panadero, E., \& Alonso-Tapia, J. (2014b). How do students self-regulate?: review of Zimmerman's cyclical model of self-regulated learning. Anales de Psicología, 30, 450-462. https://doi. org/10.6018/analesps.30.2.167221

27. Pérez, H. S., \& Gutierrez-Braojos, C. (2012). La competencia de aprender a aprender y el aprendizaje autorregulado. Editorial. Profesorado. Revista de Currículum y Formación de Profesorado, 16, 5-13

28. Riquelme, R. A., Mujica, A. D., Villalobos, M. V. P., Almeida, L. S., Herrera, I. L., Puentes, J. G., \& Strickland, B. (2012). College students' academic experiences and involvement: assessment scales adaptation and validation. Estudios pedagógicos (Valdivia), 38, 7-19. https://doi. org/10.4067/S0718-07052012000200001

29. Rivera, M., Martínez, M., Gónzález, F., \& Salazar, M. (2016). Autoeficacia, participación social y percepción de los servicios universitarios según el sexo. Revista de Psicología, 25, 1-16. https:// doi.org/10.5354/0719-0581.2016.44842

30. Rodríguez, M. S., Tinajero, C. y Páramo, M.F. (2017). Pre-entry Characteristics, Perceived Social Support, Adjustment and Academic Achievement in First-year Spanish University Students: A Path Model. The Journal of Psychology, 151, 722 - 738. https://doi.org/10.1080/00223980.2017 $\underline{1372351}$ 
31. Rosário, P., Pereira, A., Hõgemann, J., Nunes, A. R., Figueiredo, M., Núñez, J. C., . . Gaeta, M. L. (2014). Autorregulación del aprendizaje: una revisión sistemática en revistas de la base SciELO. Universitas Psychologica, 13, 781-797.

32. Ryu E. (2011). Effects of skewness and kurtosis on normal-theory based maximum likelihood test statistic in multilevel structural equation modeling. Behavior Research Methods, 43, 1066-1074. https://doi.org/10.3758/s13428-011-0115-7

33. Salanova, M., Martínez, I., \& Llorens, S. (2012). Success breeds success, especially when self-efficacy is related with an internal attribution of causality. Estudios de Psicología, 33, 151165. https://doi.org/10.1174/021093912800676420

34. Soares, A. B., Francischetto, V., Dutra, B. M., Miranda, J. M. d., Nogueira, C. C. d. C., Leme, V. R., ... Almeida, L. S. (2014). O impacto das expectativas na adaptação acadêmica dos estudantes no Ensino Superior. Psico-USF, Bragança Paulista, 19, 49-60.

35. van Rooij, E. C. M., Jansen, E. P. W. A., \& van de Grift, W. J. C. M. (2017). Secondary school students' engagement profiles and their relationship with academic adjustment and achievement in university. Learning and Individual Differences, 54, 9-19. https://doi.org/10.1016/j. lindif.2017.01.004

36. Zimmerman, B. J. (1989). A Social Cognitive View of Self-Regulated Academic Learning. Journal of Educational Psychology, 81(3), 329-339. https://doi.org/10.1037/0022-0663.81.3.329

37. Zimmerman, B. J. (2002). Becoming a self-regulated learner: an overview. Theory into Practice, 41, 64-70. https://doi.org/10.1207/s15430421tip4102__2

38. Zimmerman, B. J. (2008). Investigating self-regulation and motivation: Historical background, methodological developments, and future prospects. American Educational Research Journal, 45, 166-183. https://doi.org/10.3102/0002831207312909

39. Zulma Lanz, M. (2006). Aprendizaje autorregulado: el lugar de la cognición, la metacognición y la motivación. Estudios pedagógicos (Valdivia), 32, 121-132. https://doi.org/10.4067/S0718$\underline{07052006000200007}$

RECIBIDO: 28 de junio de 2020

MODIFICADO: 16 de diciembre de 2020

ACEPTDO: 17 de diciembre de 2020 